\title{
Supracricoid Partial Laryngectomy: The Second Coming
}

\section{Biswajyoti Hazarika}

Senior Consultant, Department of Surgical Oncology, Max Cancer Center, New Delhi, India

Correspondence: Biswajyoti Hazarika, Senior Consultant, Department of Surgical Oncology, Max Cancer Center, Max Healthcare Saket, New Delhi-110017, India, Phone: +91-9717776846, e-mail: biswa_dr@yahoo.co.in

\begin{abstract}
Early cancer of the larynx can be addressed by single modality treatment (surgery or radiotherapy) while advanced cancer larynx is addressed with multimodality treatment-surgery (total laryngectomy) + postoperative RT or concurrent chemotherapy + radiotherapy. But there is a subset of population with carcinoma larynx in whom single modality treatment with radiotherapy or conventional partial laryngectomy may not be sufficient while total laryngectomy with postoperative radiotherapy may be overkill. In this subset of population supracricoid partial laryngectomy is the perfect answer which bridges the gap between partial laryngectomy and total laryngectomy. In addition to utilizing this procedure in the post-RT residual disease scenario the upfront performance of SCPL can also avoid adjuvant RT in many instances. Supracricoid partial laryngectomy is an organ preservation procedure in the true sense that it can achieve an oncological safe margin and also provide a high quality of life.

Keywords: Supracricoid laryngectomy, Organ preservation, Cricohyoidopexy, Cricohyoidoepiglottopexy, Arytenoids, Thyroid cartilage, Cricoid cartilage, Paraglottic space, Pre-epiglottic space, Postoperative radiotherapy.
\end{abstract}

Due to recent trend of stress on the quality of life in cancer management especially that of head and neck, organ preservation both anatomical and functional, has become the norm of the day. Larynx, apart from voice production plays a stellar role in swallowing, respiration and protection of lower airway- this makes the preservation of the physiologic function of the larynx more important. For early cancer of the larynx single modality treatment either with radiation therapy (RT) or surgery (endoscopic laser surgery or partial laryngectomy) has equivalent results. Surgery (total/near total laryngectomy) followed by postoperative $\mathrm{RT}+/$ - chemotherapy or the nonsurgical protocol of concurrent chemotherapy + radiotherapy (conc. CT $+\mathrm{RT}$ ) shows equivocal result in the management of advanced cancer of the larynx. Though organ preservation protocol with concurrent use of chemotherapy and radiotherapy has showed promising results, it has its own drawbacks - i) larynx though preserved may not be functional ii) salvage surgery after failure is fraught with increased complications iii) treatment drop-outs due to toxicity iv) chance of radiation induced malignancy in young adults. There is a subset of patient population (viz. young patients, late T2, T3 with paraglottic space involvement) in whom concurrent chemotherapy and radiotherapy or total/near-total laryngectomy may be over kill while endoscopic laser surgery of vertical partial laryngectomy may not achieved oncological safety. Extended partial laryngectomies are also associated with somewhat difficult mucosal closure and require one or other form of reconstruction. Supracricoid partial laryngectomy (SCPL) provides the most favourable results in this subset of population. It provides an effective alternative to RT, supraglottic laryngectomy, total/near-total laryngectomy in selected supraglottic and glottic carcinoma.

\section{HISTORY}

The concept of supracricoid partial laryngectomy was first conceived in 1959 by two Austrian surgeons -Majer and Reider. ${ }^{1}$ After initial enthusiasm, however it fell into disrepute probably due to not so encouraging functional and oncological outcome. Through the 1970s Labayle, Piquet and Dahan ${ }^{1}$ of France modified the technique (they termed it as subtotal laryngectomy) and started promoting it in 1981 and it slowly gained acceptance in France and rest of Europe thereafter. Laccorreye combined all the modifications together and standardized the reconstruction as cricohyoidoepiglottopexy and cricohyoidopexy. ${ }^{2}$ The North Americans only adopted in 1991 and since then it has become an established procedure in several centres worldwide. ${ }^{3}$

\section{The Concept}

Extensive studies on laryngeal physiology have now established that the cricoarytenoid joint is the basic functional unit of the larynx and not the vocal cord as thought earlier. ${ }^{4}$ This forms the basis for the concept of supracricoid partial laryngectomy. 
Supracricoid laryngectomy is an organ preservation surgery in the true sense as it preserves the physiological function of the larynx (speech, swallowing, respiration and protection of lower airway) without a permanent tracheostomy along with total tumor extirpation with proper oncological safety.

There are two forms of SCPL depending on the primary site of the tumor and the type of reconstruction. The true and false vocal cords, the entire thyroid cartilage and the entire paraglottic space is resected in the procedure that is employed to address selected glottic carcinoma leaving behind the entire epiglottis, the hyoid bone, minimum one criciarytenoid joint along with its intact innervation (i.e. recurrent laryngeal nerve) and the entire cricoid cartilage. The reconstruction entails suturing the cricoid to the hyoid and the epiglottis - cricohyoidoepiglottopexy (CHEP) and hence the term SCPL +CHEP. In selected supraglottic and transglottic carcinoma in addition to the structures removed in SCPL+CHEP, the entire pre-epiglottic space and the epiglottis are resected and a pexy is made between the cricoid and the hyoid bone (CHP) and hence the term SCPL + CHP (Figs 1 to 3 ).

\section{Indications}

1. Impaired mobility or even fixity of vocal cord/cords but with mobile arytenoids in supraglottic, transglottic or glottic carcinoma forms the strongest indication. The cord fixity may be due to involvement of the thyroarytenoid muscle or involvement of the paraglottic space.

2. T1b glottic carcinoma (a horse-shoe lesion) where more than one-third of the contralateral cord is involved. These tumors can be addressed with extended vertical partial laryngectomy but requires mucosal reconstruction whereas a SCPL + CHEP obviate any need for reconstruction.

3. Supraglottic cancer with spread to the anterior commissure.

4. Thyroid cartilage involvement but without invasion of the outer perichondrium (Figs 4A and B).

\section{Contraindications}

1. Cord fixity due to cricoarytenoid joint involvement.

2. Pre-epiglottic space involvement in glottic cancer (i.e. in transglottic cancer)

3. In cancer of the supraglottic involvement of the base of tongue, vallecula and massive involvement of the pre-

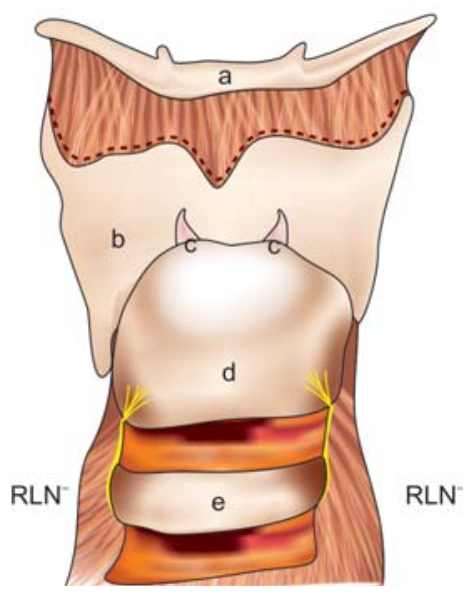

Fig. 1: Schematic diagram depicting SCPL entailing resection of entire thyroid cartilage, pre-epiglottic and paraglottic space, and preserving the hyoid bone, the entire cricoid cartilage, at least one cricoarytenoid unit and bilateral recurrent laryngeal nerve (a) hyoid, (b) thyroid cartilage, (c) arytenoids, (d) cricoid cartilage, (e) tracheal rings

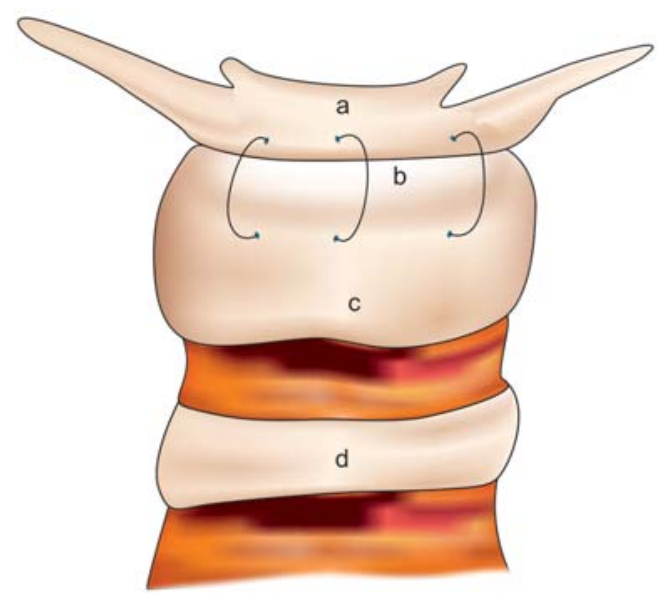

Fig. 2: Schematic diagram depicting Cricohyoidopexy - pexy is made between the hyoid bone the cricoid cartilage (a) hyoid, (b) pexy, (c) cricoid cartilage, (d) tracheal rings

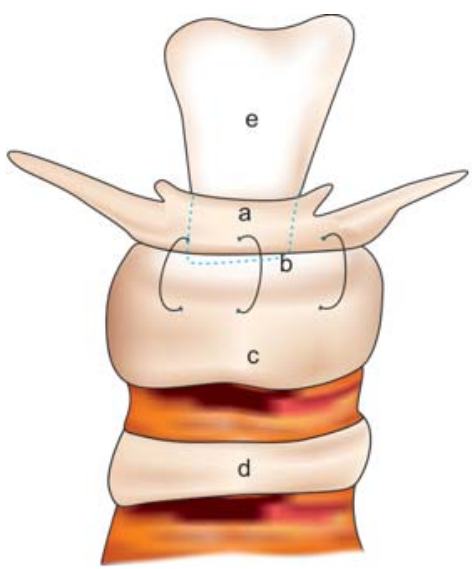

Fig. 3: Schematic diagram depicting Cricohyoidopexy-the base of the epiglottis is enclosed within the pexy between the hyoid and the cricoid cartilage (a) hyoid, (b) pexy, (c) cricoid cartilage, (d) tracheal rings, (e) epiglottis 


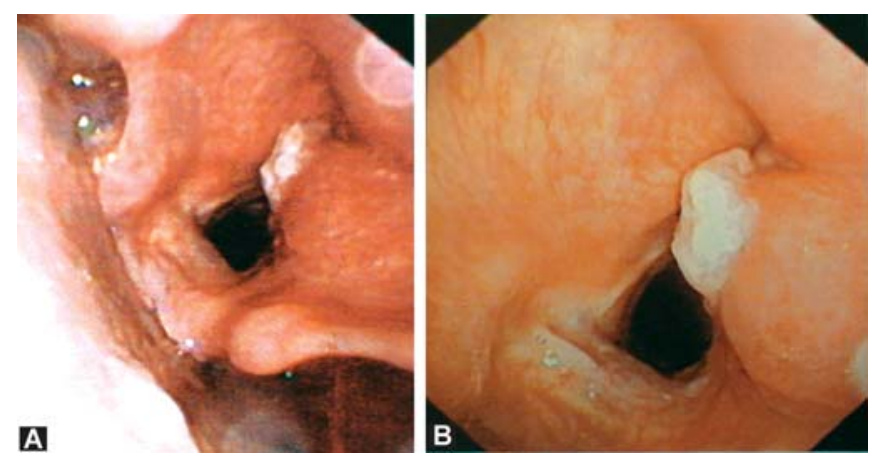

Figs 4A and B: Endoscopic view of a right sided supraglottic carcinoma with involvement of the right paraglottic space (bulge of the right false cord) with extension into the anterior commissure and the right ventricle. The right vocal cord was fixed while both the arytenoids were mobile

epiglottic space where saving of the hyoid bone is not compatible with oncological safe margin.

4. Subglottic extension (extent of tumor more that $10 \mathrm{~mm}$ anteriorly and $5 \mathrm{~mm}$ posteriorly),

5. The interarytenoid region, the postcricoid region, the bilateral pyriform sinuses should be free of disease.

6. Prior tracheostomy precludes any form of SCPL.

7. Poor pulmonary reserve is a relative contraindication.

\section{Prerequisites/Work-up}

Like any other conservation laryngectomy scrupulous case selection is the key to success for SCP+ CHEP/CHP. There's no alternative to an indirect laryngoscpy and palpation of the neck for neck nodes and extralaryngeal spread in the out patient department. Apart from this the following investigations help us to identify patients for SCPL (Fig. 5).

1. Fiberoptic laryngoscopy for a better evaluation of vocal cord movements along with that of arytenoids.

2. Direct laryngoscopy under general anesthesia apart from taking a biopsy and mapping of the lesion provides an opportunity to palpate the lesion and the surrounding structure to know the depth of the lesion. The following needs to be assessed carefully:

i. Degree of alteration of mobility of the true vocal cord

ii. Degree of alteration of mobility of the arytenoid cartilage

iii. Involvement of the anterior commissure

iv. Degree of invasion of the subglottis

v. Status of the mucosa surrounding the primary site

vi. Degree of invasion of the pre-epiglottic space

vii. Invasion of the thyroid cartilage

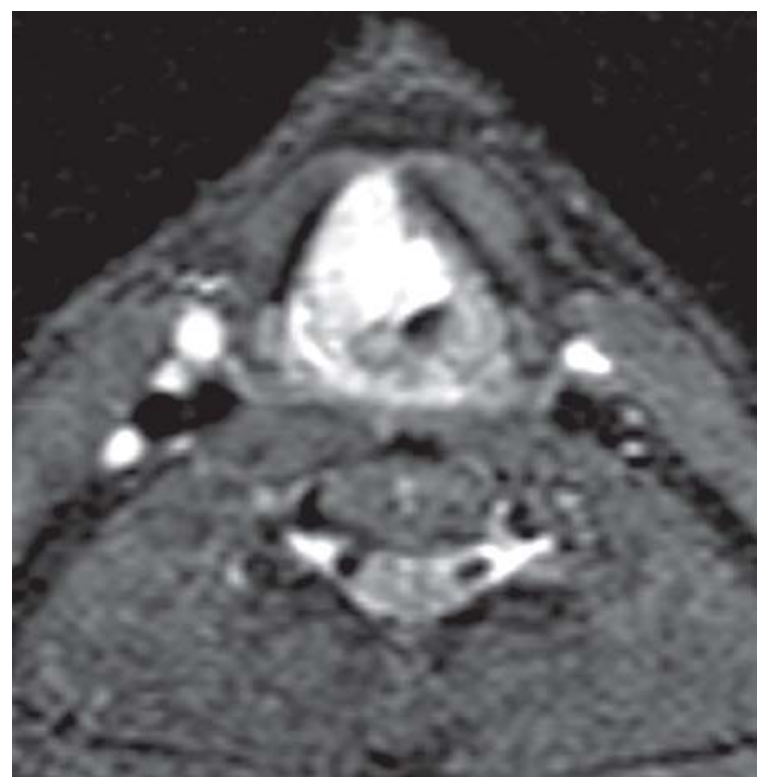

Fig. 5: MRI picture of the same patient showing right supraglottic mass with gross involvement of right paraglottic space without any gross invasion of the thyroid cartilage

3. Imaging studies viz. CT scan and/or MRI other than providing information regarding involvement of paraand pre-epiglottic space invasion also provide information in relation to status of thyroid cartilage, subglottic extension and metastatic neck nodes.

4. If a pulmonary function test is to be done then a FEV1 of $>50 \%$ is desirable. Clinical assessment rather than a pulmonary function test gives a fair about the pulmonary reserve. Chronic and inefficient cough, purulent sputum, and/or an inability to climb 2 flights of stairs without shortness of breath are strong contraindications against the use of SCPL-CHEP or SCPL-CHP.

\section{PROCEDURE}

A full description of the surgical procedure is beyond the scope in this article, but nevertheless the following key surgical points mandate a mention.

\section{Key Surgical Points}

- The superior flap needs to be elevated about $2 \mathrm{~cm}$ above the hyoid bone to prevent tethering of skin in the impaction during reconstruction.

- Infrahyoid muscles need to be resected close to the superior border of thyroid cartilage.

- Bilateral superior laryngeal nerves need to be preserved so as to maintain sensation of the remaining larynx. 


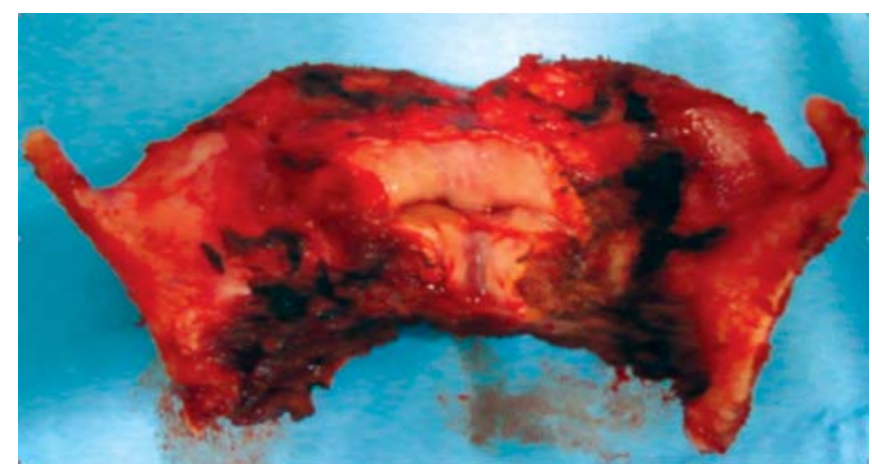

Fig. 6: SCPL specimen

- A mobile arytenoid is necessary postoperatively for laryngeal closure during deglutition and for the best possible voice quality. Subperichondrial disarticulation of the cricothyroid joint always protects the RLN where the nerve enters the larynx.

- Do not preserve the posterior third of the false and true vocal cords on the noninvolved side of the larynx. This additional tissue compromises the apposition of the arytenoid and the base of the tongue compromising the functional efficiency of the neoglottis.

- In case of hypertonia of the upper esophageal sphincter cricopharyngeal myotomy needs to be performed. But in case of history of gastroesophageal reflux a myotomy should be avoided.

- The pexy sutures should be approximately apart. Too many pexy sutures may flatten the tongue base, reducing swallowing efficiency.

- If there's fracture of the cricoid cartilage while tying the pexy sutures, the pexy should be preformed over the first two tracheal rings.

- Tracheostomy site should be made in line with the neck incision so as to facilitate replacement of the tracheostomy tube if it gets dislodged in the peri-operative period (Fig. 6).

\section{COMMENTS}

Since the early 1990s when SCPL was introduced in North America, it has gradually established itself as an organ preservation surgery for selective endolaryngeal malignancy in the world map. Till August 2010 there are more than 100 publications on various aspects of SCPL in the Englishlanguage medical literature. It's a true organ preservation surgery in the sense that it maintains the primary functions of larynx (voice, swallowing, airway protection) without compromising oncological safety. It bridges the gap between partial laryngectomy and total laryngectomy. In selected laryngeal cancer, it has proved itself as an effective alternative to radiotherapy, concomitant chemotherapy + radiotherapy organ preservation protocol, partial laryngectomy and total/near-total laryngectomy. Bridger et al, in 1996 has rightfully stated that "in the near future any patient being evaluated for total laryngectomy should be considered for the less radical supracricoid laryngectomy". ${ }^{5}$

Multiple studies have proven the supremacy of SCPL over other modalities of treatment in selected cases of laryngeal cancer in terms of local control and long term larynx preservation. ${ }^{6-10}$ Laccourreye et $\mathrm{al}^{6}$ in 1997 reported a local control rate of $98.2 \%$ at 5 years in $\mathrm{T} 1$ and $\mathrm{T} 2$ glottic carcinoma extending to anterior commissure. A 79\% 5-year survival was achieved by Chevalier and Piquett ${ }^{7}$ in a series of 61 cases of T1-T4 supraglottic cancer (ref). Laccourreye et $\mathrm{al}^{8}$ in a series of 60 cases of T3-T4 supraglottic and transglottic cancer reported 5-year actuarial survival rate of $72.7 \%$ (ref). Andrea Gallo ${ }^{9}$ et al in a long series of 253 patients with selected supraglottic and glottic cancer achieved an overall survival rate of $85.8 \%, 79.1 \%, 57.6 \%$ and $57.6 \%$ at 3-, 5-10- and 16 years respectively.

Another important fact about SCPL deserves a mention - the relative rare use of postoperative radiotherapy (Postoperative RT) after SCPL in many series. ${ }^{9-12}$ Andrea Gallo et $\mathrm{al}^{9}$ in their series were able to avoid postoperative $\mathrm{RT}$ in 10 of their cases. In the series of Brasnu D et al ${ }^{10}$ only $33.8 \%$ received postoperative RT whereas in the series of Xavier Dufour ${ }^{11}$ et al only 20.3\% patients received postoperative RT. Postoperative RT is generally reserved for patients with close/positive cut margins, multiple neck nodes and/or extracapsular spread. This avoidance of postoperative RT not only helps to reduce complications like laryngeal radionecrosis, laryngeal stenosis, stenosis of the esophageal inlet but we can actually reserve radiotherapy for the management of various metachronous second primary which is not an uncommon phenomenon in head and neck cancer.

Of late, SCPL has been able to establish itself as an organ preservation surgery even in the radiation failure scenario. Several studies like those by Andrea Gallo et al, ${ }^{9}$ Raul Pellini et $a l,{ }^{13}$ Kuauhyama Luna -Oritz et al ${ }^{14}$ Gino Marioni, ${ }^{15}$ Xavier Leon, ${ }^{16}$ to name a few stand evident to this. Tilla a few years back total laryngectomy was the only answer to radiation failure settings, whereas today a substantial number of selected patients can have the benefit of a SCPL. 
On the quality of life issue SCPL scores heavily against total/ near total laryngectomy. ${ }^{9,10,17-19}$ Though the voice in SCPL is less efficient in with respect to range, jitter, shimmer and noise: harmonics ratio, ${ }^{20,21}$ patients generally are more than happy to have their own voice and intact trachea. It allows a good control over the condition (malignancy) while having a low impact on the quality of life.

\section{SUMMARY AND CONCLUSIONS}

The organ preservation technique of supracricoid partial laryngectomy with cricohyoidopexy or cricohyoidoepiglottopexy has over the years steadily gained acceptance worldwide. Over and above achieving a local control comparable with that of total laryngectomy and/or concurrent chemotherapy and radiotherapy SCPL achieves the functional goals of speech and swallowing without creation of a permanent tracheostoma - the main detriment to the quality of life of patients undergoing total laryngectomy. Having a standard patient selection criteria and standard surgical procedure the functional outcomes of SCPL are predictable and reproducible.

The following conclusions can be drawn after a review of the spectrum of literature on SCPL

a. SCPL should be discussed with the patient whenever he/she is counseled about organ preservation protocol nonsurgical/ surgical.

b. In the radiation failure scenario, SCPL should be considered before subjecting any patient to total/neartotal laryngectomy

c. It challenges the use of adjuvant radiotherapy in laryngeal carcinoma.

\section{REFERENCES}

1. Sharad Chawla, Andrew Simon Carney. Organ preservation surgery for laryngeal cancer. Head and Neck Oncology May 2009;1:12.

2. Laccorrye H, Laccorrye O, Weinstein G, Menard M, Brasnu D. supracricoid laryngectomy with cricohyoidopexy: a partial laryngectomy procedure for selected supraglottic and transglottic carcinomas. Laryngoscope 100(7):735-41.

3. Farrag TY, Koch WM, Cummings CW, Goldenberg D, AbouJaoude PM, et al. Supracricoid laryngectomy outcomes: the John Hopkins experience. Laryngoscope 2007;117:129-32.

4. Gregory S Weinstein, Ollivier Laccourreye, Christopher Rassekh. Early glottic and supraglottic carcinoma: open supraglottic and supracricoid partial laryngectomy. Head and Neck Surgery-Otolaryngology. 4th edition (C) 2006 Lippincot Williams and Wilkins.

5. Bridger GP. Supracricoid Partial laryngectomy with cricohyoidopexy for advanced laryngeal cancer. Aust J Otolaryngol 1996;2:400-02.
6. Laccourreye O, Muscatello L, Naudo Ph, Brasnu D et al. Supracricoid partial laryngectomy with cricohyoidoepiglottopexy for 'early' glottic carcinoma classified as T1T2N0 invading the anterior commissure. Am J Otolaryngol 1997;18:385-90.

7. Chevalier D, Piquett JJ. Subtotal laryngectomy with cricohyoidopexy for Supraglottic carcinoma: review of 61 cases. Am J Surg 1994;168:472-73.

8. Laccorreye O, Brasnu D, Biacabe B, et al. Neoadjuvant chemotherapy and supracricoid partial laryngectomy with cricohyoidopexy for advanced endolaryngeal carcinoma classified as T3-T4: 5-year oncologic results. Head and Neck 1998;111:435-42.

9. Andrea Gallo, Valentina Manciocco, Marilia Simonelli et al: Supracricoid partial laryngectomy in the treatment of laryngeal cancer - Univariate and multivariate analysis of prognostic factors. Arch Otolaryngol Head and Neck Surgery July 2005;131:620-25.

10. Daniel F Brasnu. Supracricoid laryngectomy with cricohyoidopexy in the management of laryngeal carcinoma. World J Surg 2003;27:817-23.

11. Xavier Dufour, Stephane Hans, Erwan De Mones et al. Local control after supracriocid laryngectomy for "advanced" endolaryngeal squamous cell carcinoma classified as T3. Arch Otolaryngol Head and Neck Surgery, Sep 2004;130:1092-99.

12. Luc Bron, Edgard Brossard, Phillipe Monnier, Phillipe Pasche. Supracricoid partial laryngectomy with cricohyoidopexy and cricohyoidepiglottopexy for glottic and supraglottic carcinomas. Laryngoscope April 2000;110(4):627-34.

13. Raul Pellini, Barbara Pichi, Paolo Ruscito, et al. Supracricoid partial laryngectomies after radiation failure: a multi-institutional series. Head and Neck March 2008;30:372-79.

14. Kuauhyma Luna-Oritz, Philippe Pasche, Mario Tamez-Velarde and Veronica Villavicencio-Valencia. Supracricoid partial laryngectomy with cricohyoidoepiglottopexy in patients with radiation therapy failure. World J Surg Oncology 2009;7:101.

15. Gino Marioni, Rosario Marchese- Ragona, Antonio Pastore, Alberto Stafferi. The role of supracricoid laryngectomy for glottic carcinoma recurrence after radiotherapy failure: a critical review. Acta Oto-Laryngologica. Dec 2006;126(12):1245-51.

16. Xavier Leon, Montseraat Lopez, Garcia J, et al. Supracricoid laryngectomy as salvage surgery after failure of radiation therapy. Head and Neck, 2007;264:809-14.

17. Weinstein GS, El-Sawy MM, Ruiz C, et al. Laryngeal preservation with Supracricoid partial laryngectomy results in improved quality of life when compared with total laryngectomy. Laryngoscope, 2001;111(1):191-99.

18. Marguez Moyano JA, Sanchez Gutierrez R, Rldan Nogueras, et al. Supracricoid partial laryngectomy: quality of life assessment. An Otorrinolaringol Ibero Am 2007;34(1):53-56.

19. Kuauhyma Luna-Oritz, Ethel R Nunez-Valencia, Mario TamezVelarde and Martin Granados-Garcia. Quality of life and functional evaluation after supracricoid partial laryngectomy with cricohyoidoepiglottopexy in Mexican patients. Journal of Laryngology and Otology 2004;118(4):284-88.

20. Hollisinger FC, Weinstein GS, Laccourreye O. Supracricoid partial laryngectomy: an organ preservation surgery for laryngeal cancer. Curr Probl Cancer 2005;29:190-200.

21. Randal S Weber, Arlene Forastiere, David I Rosenthal, Ollivier laccourreye. Cancer, July 2005;101(2):211-19. 